\title{
PERCEPCIONES DE ADOLESCENTES DROGODEPENDIENTES FRENTE AL MODELO DE COMUNIDAD TERAPÉUTICA
}

\section{PERCEPTIONS OF ADDICTS ADOLESCENTS FRONT THERAPEUTIC COMMUNITY MODEL}

\author{
Martha Liliana Palomino Leiva ${ }^{1}$
}

\begin{abstract}
RESUMEN
Este artículo es producto de una investigación cualitativa, que tiene como propósito describir las percepciones de los adolescentes residentes en una comunidad terapéutica durante su proceso de rehabilitación. La metodología utilizada partió de la revisión de literatura relacionada con el concepto de comunidad terapéutica y su impacto en el proceso de rehabilitación en adolescentes drogodependientes. Además se recolectó información en una comunidad terapéutica colombiana, mediante la realización de entrevistas a profundidad, observaciones y un grupo de discusión.
\end{abstract}

Los resultados evidencian que la comunidad terapéutica es un espacio de encuentro personal, estructurado bajo el autocontrol y el afecto, dado que los adolescentes reconocen que este espacio favorece el fortalecimiento de vínculos afectivos, familiares y sociales a partir de la resignificación de sus problemáticas emocionales y de la adicción. También consideran que les permite la reconfiguración de sus proyectos de vida, así como el desarrollo de habilidades sociales para su integración a la comunidad de origen. Finalmente, se concluye que el modelo terapéutico - educativo posibilita la resocialización mediante procesos de identificación y formación, desarrollado por etapas, rutinas, tareas, valores y procesos de autoayuda vividos en el hogar de residencia.

Palabras clave: adicción, adolescentes, comunidad, resocialización, vivencia.

\begin{abstract}
This article is a qualitative research that has as purpose to describe the perceptions of adolescents living in a therapeutic community during their rehabilitation process. The methodology used was based on the review of literature related to the concept of therapeutic community and its impact on the rehabilitation process in adolescent drug abusers. In addition, information was collected in a therapeutic community in Colombia, by conducting in-depth interviews, observations and a discussion group.
\end{abstract}

The results show that the therapeutic community is a personal meeting space, structured under the self-control and affection, since teenagers recognize that this space also strengthens emotional ties, family and community from the re - significance of their emotional problems and addiction. They also consider that allows the reconfiguration of their life projects, and

1- Psicóloga Social Comunitaria, UNAD. Esp en Gerencia Educativa Universidad Católica de Manizales. M. A. Online Education UNAD FLORIDA. Docente ocasional - Escuela Ciencias Sociales, Artes y Humanidades ECSAH - UNAD Zona Occidente - Eje Cafetero. Colombia. E. mail: martha.palomino@unad. edu.co. Artículo de investigación. 
the development of social skills for their integration into the community of origin. Finally, it is concluded that the therapeutic model - education enables resocialization by identifying and training processes developed in stages, routines, tasks, values and self-help processes lived in the home of residence.

Key words: Adding, adolescents, community, experience, resocialization.

\section{INTRODUCCIÓN}

Las comunidades terapéuticas son de diversa índole al ser administradas por una variedad de organizaciones 0 movimientos, ya sean espirituales o de salud mental; en razón a la cual presentan diseños y enfoques distintos para el abordaje de procesos individuales, de grupo y de autoayuda. Al respecto De León reconoce que: "existen dos vertientes principales: una, orientada a la psiquiatría social que diseña tratamientos psicológicos y psiquiátricos en hospitales de salud mental, y la segunda aquella que desarrolla programas de tratamiento residencial basados en la comunidad de adictos y alcohólicos" (2004: 35), -siendo esta última la acogida en la presente investigación- puesto que los sujetos abordados se encuentran vinculados a este tipo de comunidad.

El uso de sustancias psicoactivas se ha extendido por todo el mundo, condición que moviliza el interés de las instituciones que se ocupan del fenómeno de la farmacodependencia en la búsqueda de alternativas y estrategias de prevención y tratamiento para quienes están inmersos en esta compleja problemática, que afecta a todos los grupos de la población, especialmente a los adolescentes, quienes inician su consumo a edades cada vez más tempranas.

Como es bien sabido el consumo de este tipo de sustancias tiene efectos nocivos sobre todos los ámbitos del ser humano: individual, familiary social, razón por la cual requiere un abordaje de intervención que los contemple de manera integral. Desde esta perspectiva el modelo de comunidad terapéutica (CT), avalado por diferentes organismos internacionales desde sus inicios en el año de 1975, se ha constituido en una estrategia de intervención para abordar dicha problemática.

La CT hace referencia a un espacio social construido con fines terapéuticos que posibilita la rehabilitación o el tratamiento respecto a problemas compartidos por sus residentes. Un grupo artificial duradero y residencialmente localizado (de carácter comunitario), pero con una finalidad terapéutica respecto a problemas compartidos por sus residentes - drogadicción o trastorno mental- habitualmente en proceso de rehabilitación (Sánchez, citado en Bueno \& Limiñana, 2002).

De esta definición se destacan los siguientes elementos: un grupo de 
personas que la constituyen (directivos, terapeutas, usuarios o beneficiarios), un espacio residencial localizado para el aislamiento temporal y actividades para la deshabituación y el mantenimiento de la convivencia. Al respecto Goti (2009) la define como:

una modalidad de atención residencial donde se establece una comunidad con objetivos, un sistema de control $y$ de equilibrio, $y$ un tratamiento de deshabituación o rehabilitación orientado a dar una respuesta satisfactoria o de recuperación de determinados usuarios, en este caso aquel fármaco o drogodependiente (p. 20).

Este concepto hace énfasis en un tratamiento que combina una filosofía de vida fundada en una misión institucional, en las relaciones cotidianas al compartir valores, responsabilidades, roles y una estructura metodológica que combina técnicas terapéuticas y educativas con el fin de lograr la rehabilitación o el cambio de actitud en las personas con problemas de adicción.

Molina (2011), precisa la diferencia entre los conceptos de comunidad terapéutica como modelo y comunidad terapéutica como método de rehabilitación. El modelo, o paradigma, hace referencia al marco teórico que engloba las diferentes estrategias de intervención en la que se combinan aspectos biológicos, psicológicos, socioculturales y existenciales. Son ejemplos de este modelo, el biopsicosocial, el biosanitario, la farmacoterapia, la psicoterapia, la combinación farmacoterapia psicoterapia y el holístico o mixto.

La CT como método permite el proceso de rehabilitación: normas, valores, roles, rutinas, hábitos, horarios, etc. En esta segunda acepción la estrategia elegida para favorecer el proceso de rehabilitación es la propia inclusión y vivencia en una estructura social de convivencia con una serie de normas, límites y responsabilidades establecidas; con una distribución clara en sectores, roles de trabajo y funcionamiento, con horarios, hábitos y rutinas establecidos y mantenidos en el tiempo (higiene, sueño, alimentación, tareas de la vida diaria, etc.), sumados a la vivencia en valores $y$ la transferencia cultural que supone un proceso de rehumanización de mayor o menor duración (Molina , 2011).

Al interior de la comunidad terapéutica como método, se pueden encontrar comunidadesterapéuticas no profesionales y profesionales. La comunidad terapéutica no profesional es la más tradicional y se fundamenta exclusivamente en la autoayuda (Comas, 2010); por su parte las comunidades terapéuticas profesionales son aquellas que se fundamentan en el manejo del conocimiento científico de los problemas de salud, y por tanto, requiere de profesionales idóneos, provenientes de diversas disciplinas, de acuerdo al perfil de los residentes y al tipo de comunidad terapéutica.

La Federación Latinoamericana de Comunidades Terapéuticas - FLACT (2009), también diferencia comunidades terapéuticas privadas y públicas; rurales y urbanas: religiosas y no religiosas; democráticas y jerárquicas. Existen comunidades terapéuticas para niños de la calle, para adolescentes, para menores infractores, para habitantes de la calle y para personas de cualquier sexo y edad, hombres y mujeres con problemas de adicción a las drogas. 
Sánchez (citado en Bueno \& Limiñana, (2002:57) distingue dos modelos generales de comunidad terapéutica, diferenciados por su origen, problema abordado y metodología usada: el modelo europeo denominado también democrático y el modelo americano o autoritario. El modelo europeo o democrático fue el precursor del origen de la comunidad terapéutica, fundado en la psiquiatría comunitaria por Maxwell Jones en 1941 y por Thomas Main en 1946 en el Reino Unido. Se reconoce a Jones como el gestor principal de la comunidad terapéutica en psiquiatría social (Soto, 2011).

Jones elabora las ideas básicas sobre la comunidad terapéutica $y$, en términos metodológicos, propone prácticas innovadoras como las reuniones cotidianas del personal y pacientes que contribuyeron a desmitificar la imagen autoritaria del hospital. Además pone énfasis en la autoayuda, ayuda mutua y el trabajo conjunto de todos para ayudarse a sí mismos y a los demás. El grupo funcionaría como catalizador de procesos psicológicos individuales, cumpliendo así una importante función terapéutica (Soto, 2011).

El Comité de Expertos en Salud Mental de la Organización Mundial de la Salud (OMS), reafirmó en 1957 y en 1959 -, la consideración del hospital psiquiátrico como una comunidad terapéutica en interacción con el medio social además de remarcar la relevancia de la terapia ambiental y ocupacional (Soto, 2011). Reforma que generó grandes cambios en dichas instituciones. Este modelo toma relevancia porque presenta características comunes a las comunidades terapéuticas actuales.

En 1921 surge el modelo americano o autoritario con el movimiento cristiano
Grupo de Oxford, fundado por Frank Buchman, que en el año de 1935 dio origen al movimiento de autoayuda Alcohólicos Anónimos (A.A), en la ciudad de Akron, Ohio. A partir de este movimiento surgen dos programas representativos del modelo: Synanon en 1958 y posteriormente en 1963, Daytop Village.

Synanon es un programa de tratamiento desarrollado en Santa Mónica, California, fundado por Charles Dederich (alcohólico en recuperación), para tratar drogodependientes con el fin de lograr la abstinencia sin intervención médica. Su fundamento se centró en el tejido comunitario o clima social en que las personas adictas a las drogas se ayudaban recíprocamente a enfrentar la vida de otra forma, quienes eran vistas como miembros de una comunidad y no como pacientes. Su método se basa en la confrontación para regular la vida cotidiana y facilitar la expresión de emociones (Carrizo, citado en Soto 2011: 45).

Aunque en este modelo se considera la auto-ayuda como forma de recuperación, la creencia en la capacidad individual es fundamental para curar y modificar aspectos del carácter. Esta recuperación reside principalmente en las relaciones terapéuticas con los semejantes; su objetivo se centra en la orientación psicológica para abordar el problema de la adicción, por lo cual se considera que el propio individuo es la fuente primordial en la modificación de la conducta.

A partir de estos lineamientos el Dr. Alexander Bassin y el médico psiquiatra Daniel Casriel toman algunos de los lineamientos de Synanon y, junto al Padre William B. O'Brien de la Iglesia Católica de Tuckahoe en Nueva York, fundan Daytop Lodge (Drug and Alcohol Abuse Treatment 
Programs) en Butler Manor, Staten Island, un centro de rehabilitación diseñado para 22 personas en libertad condicional masculina del sistema correccional de Brooklyn. En 1964, se transforma en Daytop Village y abre, la posibilidad de tratamiento a hombres y mujeres que buscaban ayuda voluntariamente. Inicialmente, este modelo funcionaba solo con ex adictos, bajo la noción de autoayuda. Posteriormente se vincularon profesionales de salud a los equipos terapéuticos (Soto, 2011).

En 1979, se crea en Europa el Progetto Uomo (proyecto Hombre) concebido como un sistema terapéutico de rehabilitación de drogodependientes, inspirado en las prácticas de Synanon, Daytop y la Comunidad Terapéutica Psiquiátrica de Jones (Carrizo, citado en Soto 2011: 48), con dos componentes: educativo y terapéutico. Los adictos serían personas que necesitan "reeducarse" para llevar una vida constructiva y la educación se producía siguiendo modelos de rol y recibiendo directamente la enseñanza y guía de personas rehabilitadas con anterioridad (Soto, 2011).

En los años 80, Progetto Uomo se configura como un modelo propio, diferenciándose de la escuela americana, al vincular la intervención familiar en el proceso de tratamiento, la definición de objetivos terapéuticos más personalizados y la conformación de equipos mixtos integrados por profesionales, ex adictos y voluntarios. Este modelo ha influido en propuestas similares en Europa, especialmente en España y se ha extendido a algunos países de Asia y America Latina (Soto, 2011).

En Colombia, se cuenta con las experiencias de la Comunidad Terapéutica Instituto
Amigó de Medellín, fundada en 1982 por la Congregación Religiosa de los Terciarios Capuchinos y la Fundación de Hogares Claret fundada en 1984, por iniciativa del sacerdote de la Comunidad de los Misioneros Claretianos, padre Gabriel Antonio Mejía Montoya. En esta última se aplica el modelo holístico de la CT, que en palabras de Mejía (2000), se entiende como una escuela de vida y de humanización, en la que se combina la intervención realizada por profesionales de diversas áreas (psicólogos, trabajadores sociales, abogados, médicos, etc.) y voluntarios que surgen de aquellas personas que han vivido el proceso de rehabilitación o recuperación en la misma comunidad, quienes asumen el papel de terapeuta ex adicto y que De Leon (2004) denomina paraprofesional.

La CT como modelo y método actualmente se sustenta en un espacio de convivencia, de reencuentro, de preparación y confrontación personal. Un espacio donde se reconoce al sujeto con derechos y como agente protagonista de su propio desarrollo, mediante la implementación de rutinas, la promoción de valores, la auto ayuda o la ayuda mutua ${ }^{2}$ y bases del tratamiento (Mejía, s.f.).

También en el país se destacan otros programas privados y públicos, urbanos y rurales bajo la modalidad de CT, asociados a la Corporación Colombiana de Comunidades Terapéuticas (FECCOT), los cuales cuentan con el apoyo de organizaciones sociales, religiosas y universidades nacionales y latinoamericanas. Tanto el Ministerio de la Protección Social como el Instituto Colombiano de Bienestar Familiar (ICBF), asesoran, vigilan y hacen el seguimiento

2- Estructuras grupales voluntarias y pequeñas para alcanzar un objetivo específico conformada por iguales, que se han reunido para la asistencia mutua (Katz y Bebder, 1976). 
respectivo a este tipo de comunidades, en relación a la protección de los derechos de niños, niñas y adolescentes. Estos programas se rigen por el Código de Ética de la Federación Mundial de Comunidades Terapéuticas (WFTC), y la resolución No. 0196 de 2002 del Ministerio de Salud.

Además de las comunidades terapéuticas es preciso definir que es un adolescente drogodependiente o farmacodependiente, para lo cual es necesario abordar dos conceptos: adolescencia y drogo o farmacodependencia. Para este estudio la definición de adolescencia se aborda desde el Diccionario de Psicología de Consuegra:

La adolescencia es una etapa de transición en el desarrollo humano que se da entre la niñez y la adultez, caracterizada por cambios biológicos y psicológicos. Los principales cambios físicos que se producen son un rápido aumento de estatura y peso, cambios en las proporciones, en la forma corporal y el desarrollo de características sexuales. Los psicológicos más importantes son los signos de autonomía; distanciamiento de los adultos que hasta ahora influían de manera importante en su vida, excesiva preocupación por la apariencia física, egocentrismo, búsqueda de identificación con su grupo de pares, rebeldía, entre otras. Se considera que su comienzo se da en la pubertad aproximadamente entre los 12 años y suele extenderse hasta el comienzo de los 20 años... (2011: 5).

Desde esta perspectiva el adolescente se considera una persona vulnerable, tanto al consumo de sustancias psicoactivas, como a la violencia y al contagio de enfermedades de transmisión sexual como el Síndrome de Inmunodeficiencia Adquirida, entre otras, porque apenas está construyendo su personalidad e intenta explorar el mundo que le rodea, sin medir sus consecuencias. Además se enfrenta a grandes desafíos sociales, emocionales y académicos presentes en su contexto próximo principal de referencia (familia y comunidad). Es allí donde construye su identidad, proceso y contexto que por diversas circunstancias predispone al consumo y abuso de sustancias psicoactivas, sumado esto a aspectos genéticos y psicológicos.

El concepto de drogo o farmacodependencia se aborda desde un enfoque psicoanalítico, y se considera como un efecto de otras situaciones de vida personal, familiar, social o laboral, que motiva a una persona a consumir una sustancia, de la cual se hace dependiente $y$, le produce efectos perjudiciales para su salud.

Al respecto Bernal expresa:

Para el psicoanálisis la toxicomanía no constituye una categoría clínica; se trata más bien de un síntoma, y por lo tanto, no es la causa, sino una consecuencia. Esto es muy importante subrayarlo: la adicción a una droga no es el problema, sino la consecuencia de algún problema del sujeto. En términos generales se puede decir que la droga es una respuesta, una "solución» a un malestar particular del sujeto. La droga no es la causa de la adicción, como suele pensarse; la droga es la consecuencia. Sobre la causa hay una incógnita, por eso es a la causa a la que hay que apuntar (2011:4).

Es importante mencionar que el fenómeno de la drogo o farmodependencia se relaciona en el ámbito mundial, con un problema de salud pública, por la magnitud de los daños que se ocasionan a nivel individual y social tal como lo ha 
reconocido la Organización Mundial de la Salud (OMS) y recientemente el Congreso de la República de Colombia.

Para comprender este fenómeno de una manera holística, se introduce la dimensión del contexto sociocultural dado que su explicación se encuentra en el mundo social, en el acceso diferencial a diversas variables, en los patrones culturales, en situaciones socio-familiares $y$ en las características psicológicas individuales. Además no tiene barreras de clase social, edad o sexo; cada grupo social dependiendo de su clase, cultura - contexto presenta acercamientos particulares a las sustancias psicoactivas.

El Instituto Nacional sobre el abuso de Drogas (NIDA) (2004), destaca entre los factores de riesgo para la farmacodependencia, la conducta agresiva, la falta de supervisión de padres, el abuso de sustancias, la disponibilidad de estas y la pobreza. EI NIDA también considera la presión de los compañeros que abusan de las mismas, durante los años de la adolescencia.

Estos factores hacen que el abordaje de la adicción y la interpretación contextual de los adolescentes sea un proceso complejo, puesto que son muchos los factores y perspectivas que modulan sus problemas, realidades intersubjetivas y contextos, así como son diversos los actores involucrados. Sin embargo, el modelo de $C T$, se constituye en una alternativa terapéutica exitosa tal como lo demostró Frank Buchman y Maxwell Jones.

Pérez Del Río (2011), observó al primer mes y a los seis meses del tratamiento terapéutico - educativo, cambios en la actitud frente a las drogas, disminución en el consumo y la agresividad, aumento de la confianza y el reconocimiento de sí mismo, gracias a la terapia y las relaciones de autoayuda presentes en la CT.

Desde este marco de referencia se buscó comprender el significado que los adolescentes le dan al modelo de Comunidad Terapéutica, como estrategia para superar sus problemas de adicción o drogodependencia, insertos en un escenariodesocializacióny reconstrucción, dado a partir de la interacción y el aprendizaje social, el sentido existencial y las motivaciones personales. Por ello se realizó una investigación cualitativa que utilizó el método fenomenológico, el cual centra su estudio en las realidades vivenciadas por las personas, en este caso, los adolescentes residentes de una comunidad terapéutica colombiana.

\section{METODOLOGÍA}

\section{Método}

La investigación se realizó bajo los parámetros del enfoque histórico hermenéutico y del método fenomenológico, que permitió conocer desde las voces de los residentes, sus vivencias, significados y valoraciones frente al modelo de tratamiento de la comunidad terapéutica abordado, así como la expresión de sus actitudes, pensamientos, sentimientos y comportamientos, sin ser juzgados por su condición (Martínez, citado en Aristizabal, 2008: 70).

\section{Participantes}

En la investigación participaron once (11) adolescentes, seis hombres y cinco mujeres, residentes en dos programas de la comunidad terapéutica Hogares Claret en Colombia, bajo el modelo 
terapéutico - educativo en su segunda fase de implementación durante los años 2009 y 2010. Para su selección se tuvieron en cuenta los siguientes criterios: a) Interés y disposición para participar en la investigación. b) Adolescentes hombres y mujeres residentes entre los 14 y 18 años de edad. c) Residentes que no hubieran interrumpido su proceso terapéutico, con un mínimo de seis (6) meses de vinculación a la institución.

También participó el equipo técnico de los programas terapéuticos, integrado por dos trabajadores sociales, dos psicólogos, tres operadores, y un director regional. La participación se realizó a través de grupos de discusión.

\section{Técnicas de recolección de información}

La información se recolectó a través de una entrevista a profundidad, observación y un grupo de discusión. Por medio de la entrevista, la observación y el relato se describieron las vivencias, prácticas, sentimientos, representaciones, interacciones y marcos de referencia con relación al modelo terapéutico y el contexto de procedencia de los residentes de los programas.

El grupo de discusión realizado con el equipo técnico posibilitó el conocimiento de la estructura institucional, los componentes del programa y la socialización de la investigación.

La información obtenida fue analizada desde el enfoque interpretativo descriptivo, abordado desde la narrativa descriptiva y la re-significación de la realidad vivida por los usuarios. En el análisis se utilizó la inducción analítica a fin de identificar marcos de referencia, sistemas de valoración, lógicas de significación personal y social construidas a partir del lenguaje, en relación al modelo de comunidad terapéutica.

\section{Procedimiento}

En el desarrollo de la investigación se tuvieron encuenta los siguientes momentos: a) Conformación del equipo de trabajo y alistamiento: en este momento se capacitó a los miembros del equipo, se diseñaron los instrumentos de recolección de información y se socializó el proyecto al equipo técnico y a los residentes de la comunidad terapéutica. b) Recolección y tabulación de la información: se desarrollaron las entrevistas, las observaciones, los grupos de discusión; se transcribieron las entrevistas, se diligenciaron los relatos y los diarios de campo. c) Análisis e interpretación de la información: se realizó la relectura de los relatos y diarios de campo, se codificaron, y se elaboraron ideas analíticas y unidades de significado. Finalmente se interpretaron sus resultados y se establecieron conclusiones.

\section{PRESENTACIÓN DE RESULTADOS Y DISCUSIÓN}

Los resultados se presentan organizados a través de categorías emergentes, clasificados a través de la búsqueda de relaciones entre las características y procesos que conforman la CT y las percepciones de los adolescentes frente a su estructura, función y su interrelación con el cambio de actitud y comportamiento.

\section{Significados frente al modelo de comunidad terapéutica}

La mayoría de los adolescentes entrevistados se refieren a la CT, como un hogar de encuentro y de formación; un lugar de descanso y esparcimiento; 
un proceso de socialización donde se aprende a convivir; un lugar bonito, extraño pero que con el transcurrir de los días encuentran normas y límites, valores, autoridad, estrategias terapéuticas y educativas. Al respecto un participante expresa: '

'esta comunidad terapéutica está en el convivir, convivir con varias personas que son iguales a mí, un proceso que te ayuda bastante en lo sentimental, en la convivencia, y en la exigencia. Pero desde esta comunidad se aprende a convivir".

Otros residentes manifiestan:

"me lo imaginaba como una cárcel o como una correccional de menores, un hospital psiquiátrico... Sin embargo, con el transcurrir del tiempo me di cuenta que la comunidad es totalmente diferente".

Estas expresiones de los adolescentes evidencian que encuentran allí un espacio de reconocimiento, de comprensión, de diálogo, orientación y sobre todo de crecimiento personal y espiritual.

Tales expresiones sustentan la idea que la comunidad terapéutica es un espacio de interacción y aprendizaje social donde los residentes se encuentran con pares, que finalmente se constituyen en modelos de vida, de cambio personal y de éxito para otros. Espacio donde se desarrollan acciones y estrategias terapéuticas, y donde los participantes se constituyen en mediadores para producir cambios terapéuticos y educaciones (Goti, 2003).

Al momento del ingreso y acogida los residentes manifiestan que la principal motivación es la necesidad de recuperar su familia, el re-establecimiento de las relaciones interpersonales, especialmente lo relacionado con el sufrimiento de su progenitora y la recuperación de sus vidas. También destacan la necesidad de protección frente a las situaciones de riesgo que encuentran en la calle por los actos delictivos que alguna vez cometieron, tal como lo expresan los siguientes participantes:

“...No, como le digo, yo ya había hecho un proceso acá hace seis años, me enfermé, entonces yo me fui, pero regresé.... Me cansé, me estaba destruyendo allá fuera. Tenía muchos problemas con mi familia, muchos problemas con mi mamá, con toda la gente de la casa, problemas con la calle, y yo no me aguanté eso y me vine a internar".

"Pues se me dificultó mucho, nunca pensé en internarme y menos exactamente en diciembre, yo le decía a mi mamá que no, que yo no me iba internar, que yo no necesitaba de esto, que yo podía cambiar solo. Duré seis meses esperando el cupo, y no sé de un momento decidí venirme, porque estaba más seguro acá que en la calle".

Según lo anterior, el contexto genera circunstancias que determinan el presente y futuro de los adolescentes. De allí la importancia de aprender a vivir en comunidad, proceso que desde la CT se desarrolla mediante el reconocimiento de sí mismo; la re-significación de pensamientos, sentimientos, auto percepciones y hábitos, así como el desarrollo de habilidades sociales para su reinserción nuevamente a la sociedad.

Algunos, y en especial las mujeres, destacan la necesidad de mejorar su situación física y emocional, el deseo de cambiar y construir una vida diferente. Sin embargo, lo que más pesa al momento del ingreso es la difícil situación con la familia, tal como lo expresa uno de los participantes: 
“.. lo que me motivó fue que yo iba a recuperar mi familia, a tener menos problemas. $Y$ que daría ejemplo a mis hermanos. Por eso fue que yo entré acá".

Durante el proceso se presentan dificultades relacionadas con el deseo de no estar en la institución o por el retiro debido a la presencia de alguna enfermedad. Lo que implica participar con la presencia de su familia. Además algunos mencionan que se dificulta el ingreso en temporadas especiales como las fiestas decembrinas y ferias. Por ello postergan el ingreso o si lo hacen, desertan fácilmente.

Para otros, este proceso es muy fácil y desde su llegada manifiestan estar muy dispuestos, quizás por la motivación y el deseo de superación con que ingresan al programa. Por ejemplo: uno de los participantes manifiesta:

"...en mi vida ya he perdido a mi papá y perdí a mi hermano por el mismo cuento de las drogas, y pues no... quiero salir, quiero salir adelante".

En la etapa de ingreso y acogida los adolescentes reflejan poca motivación e iniciativa personal para cambiar por sí mismos. Simplemente piensan que su problema de adicción no es tan grave. Situación vista como normal para ellos por la falta de significado y de expectativas frente a la vida. Durante estos primeros días manifiestan que sus vivencias son realmente difíciles, afectando su proceso de adaptación.

Se destaca que la CT como modelo y como método se constituye en una estrategia de intervención terapéutica y educativa que genera cambio en las actitudes y comportamientos negativos de los adolescentes que deciden resocializarse. Se encuentra coincidencia con el modelo holístico o mixto y el método profesional que vincula la experiencia del ex adicto y la autoayuda abordado por Molina (2011) y Comas (2010), puesto que se constituyen en referentes básicos para la construcción de la comunidad, un espacio de convivencia que posibilita el reencuentro, la reeducación, la prevención y la protección de los adolescentes.

Sin embargo, es primordial que el residente cuente con deseo, voluntad y compromiso para vincularse a la CT puesto que muchos no alcanzan el objetivo en la fase inicial del tratamiento. Esto hace que sea necesario alcanzar un alto grado de motivación y participación del residente con la perspectiva de mantenerlo a mediano y largo plazo (Amaro, Chana \& Martin, 2010).

Sin esta empatía y demanda explícita del sujeto, no se logra éxito en su rehabilitación. Igualmente aunque se evidencia ruptura con su entorno familiar y social, se requieren establecer vínculos y mecanismos que posibiliten su transformación. Así como, mejoramiento en la comunicación, las relaciones sociales y la resolución de conflictos emocionales buscando evitar recaídas.

\section{Las normas institucionales como referentes de comportamiento social}

Durante la etapa de progreso lo que más resienten los residentes es la normatividad institucional expresada en rutinas y horarios que buscan un reordenamiento sociocultural y emocional con el propósito de generar autocontrol en su movilización al interior del hogar, dado que en la familia y en el contexto sociocultural, por lo general se carece de autoridad y de regulación. Pero con el tiempo ellos mismos reconocen y comienzan a entender las razones de tales normas y a darles valor, como lo 
expresan los siguientes participantes:

"No pues al principio me parecía maluco, pues venia de ser una persona ingobernable, no me interesaba nada, y simplemente hacia lo que yo quería, y llegué a un mundo de reglas y poco a poco me fui acoplando".

"Lo más difícil es el cumplimiento de muchas normas porque son normas que uno no conoce... Difícil como el no poderse comunicar con sus compañeros de varias etapas, porque cuando llegué aquí no se podía hablar sino con las más altas etapas. Esa fue una norma que yo decía tan boba pero ahora uno ve que la norma si sirve".

Los adolescentes manifiestan que en la primera y segunda fase no les gusta la presencia de la norma. Algunos las califican como una "bobada" y otros, exagerada, por ejemplo: no colocarse manillas o alhajas, las filas que deben hacer para todo procedimiento, no comunicarse con los compañeros de acogida, el tener que asumir las fallas de los demás, la levantada temprano y las rutinas que deben seguir a lo largo del día, pero como se mencionó anteriormente, al final reconocen la importancia de éstas en su proceso de adaptación y formación.

Es claro advertir que la vida social como en cualquier otra comunidad es estructurada y planificada, que permite al residente asumir responsabilidades pero a la vez evaluar su progreso individual. En este marco las palabras o acciones de crítica, corrección, castigo, afirmación o apoyo. Así, los cambios en las estructuras, privilegios y sanciones disciplinarias se orientan a que el residente alcance las expectativas de la comunidad (Soto, 2011).

En la CT muchos manifiestan que ante esta normatividad sienten deseos 0 intentos de abandono pero la familia y los educadores (ex adictos rehabilitados), se constituyen en un pilar fundamental para no desistir y continuar con el proceso, sobre todo cuando la familia está en permanente contacto y se involucra en el proceso terapéutico del residente. Estos le dan importancia al diálogo y al acompañamiento de sus familiares, así como la motivación que reciben de sus compañeros mayores y demás miembros del equipo técnico, en razón al cariño y respeto que les ofrecen, el afecto y la comprensión de su realidad.

Los residentes recién llegados destacan que se les dificulta establecer una buena comunicación con los compañeros de la etapa de elaboración, ya que ellos representan el cumplimiento de las normas y ponen la disciplina en el hogar, son vistos como rivales, como intrusos o personas que les molestan a toda hora, sin una ayuda educativa. Pero según De Leon (2004) a lo largo del proceso se generan cambios en su percepción, porque ésta se maneja a partir de privilegios y sanciones que se constituyen en un sistema de autorregulación y desafío, al interior de la comunidad.

Esto, porque en palabras de Soto (2011), los privilegios se constituyen en refuerzos positivos que favorecen las conductas adaptativas de los residentes y las sanciones se dirigen a eliminar conductas que se no se ajustan a las expectativas de la comunidad. Por ello durante el proceso de rehabilitación la norma, la rutina y la autoridad se constituyen en una herramienta y en un referente simbólico de comportamiento social, sin que sea impuesto en la comunidad terapéutica.

A esto se suma el planteamiento de Lapassaden (citado en Pinzón \& Sánchez, 2005: 23), la institución es "un conjunto de normas, pautas y actitudes agrupadas 
alrededor de roles y funciones sociales; un grupo social aceptado (escuela, empresa, sindicato); un sistema de reglas que determinan la vida de estos grupos". Siendo la CT una institución que vincula una organización social, un régimen de actividades, un proceso y un equipo terapéutico - educativo.

Es importante comentar que las normas, sanciones y medidas disciplinarias en esta CT están relacionadas con el código de Ética de la Federación Mundial de Comunidades Terapéuticas (WFTC) y la Federación Latinoamericana de Comunidades Terapéuticas (FLACT), puesto que así como se promueven deberes también se promueven derechos de los usuarios.

Entre ellos se destacan el apoyo constante, la dignidad, el respeto, la salud y la seguridad, conocer los fundamentos filosóficos, principios y normas de la CT, mantener comunicación con personas significativas, excepto cuando se indique lo contrario por razones técnicas, estar protegido y abandonar el programa en cualquier momento. Esto hace que sea acreditada y aceptada para proveer los servicios de tratamiento dentro de un marco legislativo y administrativo.

\section{Relaciones, vínculos y modelos de referencia en la comunidad terapéutica}

Los residentes manifiestan que psicológicamente ingresan preparados para defenderse de agresiones de compañeros o del equipo terapéutico, como lo expresa el siguiente participante:

"...me los imaginaba de otra forma, no sé que había gente así como yo, consumidora, me imaginaba que era gente como agresiva, gente que le gustaba buscar problemas, pleitos, así, entonces, yo dije: ah bueno, yo me voy para allá, y si tengo problemas, pues me toca resolverlos, porque no me voy a dejar de nadie, ni de nada. Pero cuando llegué acá todo fue diferente, había compañeros que no peleaban, que ante todo eran compañeros, y se llevaban bien y todo, y ahí fue que aprendí...".

En palabras de De Leon (2004), lo anterior indica que esta comunidad propicia un manejo emocional adecuado y además posibilita el desarrollo de habilidades sociales para desarrollar constructivamente sus emociones y sentimientos, o como ellos mismos dicen: manejarse bien. Proceso dado a partir del ejercicio de autocontrol, la tolerancia y el privilegio.

Las primeras relaciones significativas que se establecen en el hogar son con el hermano mayor (un residente más antiguo). Esta figura es para los residentes un referente especialmente de vivencia, intercambio y modelo; le siguen el educador que los recibe y finalmente las relaciones con los amigos y compañeros de habitación, con quienes comparten durante sus primeros meses en el programa.

Estos se constituyen en modelos y guías permanentes en su proceso de recuperación. Sobre todo, resaltan la importancia de la figura del hermano mayor (residente más antiguo), quien los acoge y los guía durante sus primeros días. Interacción que rompe con el imaginario inicial del trato agresivo y mal tratante que esperaban encontrar. Por el contrario, se encuentran con un espacio lleno de amor, cariño y de acogida.

En la comunidad terapéutica los educadores (ex adictos rehabilitados) también son grandes modelos de identificación para 
los residentes. Esto porque han vivido similares antecedentes y problemas de consumo, tiene experiencia en el manejo de dicha problemática de una manera más comprensiva y abierta, característica que otros modelos no logran establecer en la formación, ni en el crecimiento personal.

Según Ágüelo Muñoz (2004) y Olivar (2010), el rol del educador social juega un papel fundamental, siendo un referente para el usuario (sujeto residente). Esto porque mantienen una relación directa en la que establece procesos comunicativos, educativos y relaciones interpersonales para su adaptación y ayuda al interior de la CT como para el cambio personal. Por ello, su postura debe ser activa, mostrar autoridad, responsabilidad y empatía. Su intervención se hace en la vida cotidiana de la CT en distintos momentos y durante la socialización del residente.

En cuanto al afecto el género masculino expresa un sentimiento superfluo en los lazos de amistad. Manifiestan ser muy superficiales en el contacto con el otro, lo que los diferencia del género femenino, pues las mujeres son más expresivas y mencionan algunas características derivadas de su regular relación con sus compañeros. Se observa que todos los residentes tienen por lo menos una experiencia significativa en la comunidad, con relación a la amistad y los vínculos afectivos que se establecen dados por los roles y testimonios de vida de los terapeutas como modelos de vida, motivación y experiencia para el crecimiento y la superación personal.

\section{Rutinas, vivencias cotidianas y valores de interacción}

Las rutinas se constituyen en el régimen diario y variado de vida al interior de la comunidad, orientadas por horarios $y$ escenarios de actuación. Entre ellas se mencionan las reuniones por la mañana, al medio día y por la noche, tareas laborales o del hogar, grupos terapéuticos, seminarios, tiempo personal, y de recreo.

Al explorar los espacios terapéuticos los adolescentes residentes destacan y le dan un valor especial al encuentro de la mañana con su expresión de sentimientos, las terapias de grupo, la terapia de confrontación, los espacios individuales con psicología y la tutoría, al igual que los espacios de trabajo con la familia, la maratón, el aquí y ahora.

Apellániz, Gómez, Moreno y Muñiz (2010), expresan que en la intervención educativo - terapéutica son esenciales varios elementos de la CT el cual permiten la adaptación y resocialización del adolescente drogodependiente, entre ellos, la reproducción y organización de la vida cotidiana de aquellos puesto que no tienen establecidos unos horarios o rutinas diarias y no existe una figura de vínculo adulto, presente en este tiempo, que ejerza de modelo para ellos.

Estas rutinas y vivencias se desarrollan a lo largo del proceso terapéutico, organizado por etapas buscando lograr objetivos a corto, mediano y largo plazo. Proceso que distribuye el aprendizaje, el seguimiento y la evaluación tanto del personal como de los mismos residentes. Así mismo, señalan el retraso, la gratificación, finalización de tareas y el alcance de metas.

Durante estas etapas se realizan actividades terapéuticas tales como las reuniones, seminarios, encuentros de grupo, intervenciones clínicas, talleres con familiares, salidas, encuentros lúdicos - recreativos, trabajo y asistencia a algún programa educativo externo a las instalaciones, con el fin de acompañar a 
los adolescentes en la construcción de sus proyectos de vida a partir de procesos de formación integral.

Las vivencias de los adolescentes a partir de estas rutinas posibilitan el encuentro consigo mismo, con sus pares y con su familia, permite la confrontación y práctica de valores de cooperación, solidaridad y el trabajo. También favorecen el desarrollo de vínculos dados a través de los espacios de encuentro personal y grupal donde se manifiesta el amor e interés consigo mismo y por los otros; se establecen acuerdos, compromiso de vida y de grupo así como se desarrolla el liderazgo con la comunidad.

Igualmente etapas y rutinas ofrecen un contexto que satisface las necesidades personales básicas y se establecen relaciones de calidad con figuras o modelos que favorecen seguridad, estructuración de la personalidad y desarrollo de la autonomía personal (Apellániz et al., 2010). Aquí los residentes destacan la orientación de sus pares (hermanos mayores, que ya han vivido algunas fases), y al educador social como modelos de identificación y modelamiento.

Por ello, como dice Comas (2010) el aprendizaje social y la intervención educativa se constituye en un elemento integrador $y$ en el adolescente le permite un crecimiento significativo por estar en plena etapa de desarrollo. Esta integración bajo la supervisión, acompañamiento y ayuda del adulto, pares guías y la intensificación de procesos terapéuticos establece condiciones de convivencia y les invita a compartir, negociar y colaborar como en cualquier otra comunidad.

En cuanto a los valores, conductas ideales que el equipo terapéutico promueve, procedentes de las normas de la CT con el propósito de generar autorregulación, diálogo y conciencia, los adolescentes señalan como relevantes la responsabilidad, el respeto, la amabilidad, la fidelidad, el servicio, la ayuda mutua, la espiritualidad, la humildad, la honestidad, la solidaridad, y el amor responsable como los más significativos de la interacción cotidiana. También recalcan la amistad y la espiritualidad al tener una buena relación consigo mismo, con los otros y con Dios. A continuación se presentan los siguientes fragmentos:

"No, pues el respeto, yo antes no respetaba a nadie. Yo pasaba por encima de todo el mundo, así fuera educador. $Y$ no, ahora ya es diferente, así como llegarle a la gente".

"Yo veo que si nos sirven porque una persona sin valores es una persona que no tiene sentimientos, entonces para mi tener valores es una persona que está bien; la persona que le gusta ser responsable y ayudar a las demás personas".

Estos adolescentes también manifiestan que existen valores difíciles de practicar. Tales como el amor hacia las personas, la honestidad, la comunicación adecuada y la tolerancia. Al respecto, uno de los participantes expresa:

"con el amor hacia las personas porque soy desconfiado, eso me falta, tengo que trabajar en esto porque no sé por qué soy así.

De igual forma reconocen que los valores son importantes para su vida, son una gran herramienta para enfrentarse con su realidad y su vida social posterior. Manifiestan que los adquirieron durante todo su proceso terapéutico y que además serán de gran ayuda para su vida futura. En efecto, desde la perspectiva de Comas (2010), el sistema de valores se constituye 
en una nueva cultura significativa, que incluye ciertas actitudes, que a su vez influyen en los comportamientos habituales, el cual se construyen de manera bidireccional.

Entre los valores a fortalecer, los adolescentes indican los siguientes: la humildad, la honestidad, el trabajo en equipo, la exigencia, un vocabulario adecuado y la transparencia. De hecho, un participante considera que:

"Se trabaja el valor de la humildad, la honestidad, la responsabilidad......el valor de ser honesto es como el que más se resalta. La honestidad y la humildad se dan para poder hacer las cosas bien. Para dejar de ser arrogante, para convivir mejor. Por eso creo que se deben mejorar".

Es importante resaltar que los valores comentados por los adolescentes son de tipo moral, laico y democrático. Entre ellos se infieren tres valores básicos del trabajo socioeducativo y de actuación en la CT abordado por Olivar (2010): autonomía, cooperatividad y sentido de pertenencia. En la autonomía se asocia la responsabilidad, en la cooperatividad el respeto, la amabilidad, la ayuda mutua, la tolerancia y la solidaridad, entre otros; en cuanto al sentido de pertenencia se destaca la participación y la inclusión, poco identificadas por los adolescentes.

\section{Contribuciones de la comunidad terapéutica al adolescente residente}

Del análisis realizado se identifican diversas contribuciones de la CT en el estilo de vida y la identidad del adolescente, resultado de la adaptación asistencial, al vincular un modelo educativo terapéutico en su proceso de desarrollo, logrando así la deshabituación del uso y abuso de las sustancias psicoactivas.
Estas contribuciones que a continuación se describen surgen de un mismo espacio compartido con incidencia en diversos niveles o áreas de desarrollo humano (personal, espiritual, familiar y social).

A nivel personal se fortalece la motivación, la autoestima, la identidad, el sentido de vida; los adolescentes recuperan la confianza en sí mismos. Se refleja el deseo de cambio y superación constante. Se desarrollan habilidades emocionales que posibilitan la escucha asertiva, el razonamiento, la toma de decisiones y el afrontamiento de conflictos. Es decir, la CT como sistema y estructura genera un proceso de empoderamiento personal humanizado, tal como lo describe Molina (2011).

En este proceso un referente fundamental de superación es el proyecto de vida siendo este el camino para la re-significación de sus problemáticas y el sentido existencial, proceso que se afianza en la etapa de consolidación y se pone en marcha en las dos últimas etapas del proceso: egreso y desprendimiento gradual y la etapa de seguimiento. En palabras de Comas (2010), esto hace que el adolescente construya un proyecto de vida alternativo fuera de la $C T$.

La espiritualidad se constituye en un pilar fundamental en la vida de los adolescentes, impulsa actitudes y valores trascendentales tales como la solidaridad y la honestidad, acentúa la libertad humana y asumen la vivencia de la oración, cimiento para su propio crecimiento. Desde el discurso se identifica que han recuperado el sentido y la importancia de un Dios supremo, así como la vivencia de rituales religiosos, como el de ir a Misa y practicar los sacramentos.

Igualmente esta CT añade la meditación 
trascendental y la pedagogía Scout como elementos reeducativos necesarios para que el adicto, en interacción con el grupo, construya y lleve a la práctica su proyecto de vida, teniendo en cuenta que él es agente y protagonista de su propio cambio (Fundación Hogares Claret, 2012).

A nivel familiar se restituyen los vínculos afectivos, se fortalece la relación con los más allegados (padres, hermanos, tíos) a través de la comunicación y el compartir espacios y experiencias. La madre se constituye en su principal referente, así mismo les motivan y acompañan de forma permanente en las adversidades de la vida. En palabras de Comas (2010) y Molina (2011), dentro de la CT expresiones como "la Familia" y "el Hermano", son fundamentales, lo que las convierten en una familia sustituta.

Esto porque la familia como estructura social primaria presenta un mal funcionamiento y por ende, vivencia la exclusión emocional, siendo ésta una de las causas de las adicciones en los adolescentes. Así, dentro de este análisis el sistema de relaciones sociales es fundamental pues crea lazos y afectos, que como en cualquier otra estructura de parentesco satisface las necesidades afectivas, emocionales y entrena en habilidades para cualquier espacio de interacción social.

Razón por la cual, la familia es percibida como unidad de tratamiento, puesto que se considera una posibilidad de cambio durante todo el proceso de rehabilitación, siendo esta un apoyo fundamental para la identidad y el bienestar de los miembros que la integran, en especial para los adolescentes. De esta manera la familia se vincula desde el momento del ingreso hasta la salida del proceso.
En este modelo, las técnicas y las intervenciones que los adolescentes residentes identifican de manera significativa son la reelaboración de la vivencia de la historia familiar, la entrevista familiar, la terapia familiar y las salidas programadas con la familia. Esto porque manifiestan mejora en la comunicación, la figura de autoridad de los padres, se establecen normas y límites que posibilitan la convivencia familiar, se disminuyen los conflictos y los malos tratos entre los miembros.

En lo social se mejoran las relaciones interpersonales y el vínculo afectivo con los amigos. Se desarrollan valores, responsabilidades y competencias sociales positivas al vincularse el grupo de iguales como herramienta pedagógica dentro del contexto residencial, el cual posibilita la integración en el entorno comunitario posteriormente. En el proceso metodológico propuesto por Mejía (2000), el grupo de pares se constituye en un elemento fundamental del tratamiento, al permitir su integración como red de apoyo social a lo largo del proceso pedagógico terapéutico.

En lo educativo se presenta la iniciativa e interés por vincularse al colegio o laboralmente a un oficio. En el hogar, las mujeres se capacitan en estética y belleza y los hombres en sistemas, ebanistería y mecánica. Esto posibilita formación, disciplina, concentración y responsabilidad consigo mismo y con los demás, aspectos fundamentales para la construcción de sus proyectos de vida.

En este proceso de integración yaprendizaje social se generan motivaciones, demandas, responsabilidades, roles e interacciones sociales, que a partir del hábito, la auto reflexión, el autocontrol, el trabajo, el ejemplo cotidiano y el manejo de recursos, los residentes logran cambios 
y nuevos estilos de vida, constituyéndose en un escenario continuo de identidad y aprendizaje social; es allí donde la CT se constituye en una micro sociedad para que los residentes pueden integrarse socialmente (De Leon, 2004).

En cuanto a la eficiencia de este modelo de comunidad terapéutica en la población residente adolescente, se observan cambios en la deshabituación, el reconocimiento y la valoración de sí mismos, la restitución de sus relaciones familiares, la resignificacion de valores y el sentido de vida a partir de la espiritualidad, la meditación transcendental y la educación integral. Sin embargo, se advierte que aún no se encuentran evidencias empíricas que sustenten la efectividad del modelo en todos y cada uno de los residentes por la misma complejidad del fenómeno.

Sin embargo, Molina (2011) cuando hace referencia a la CT como método, menciona que esta favorece el proceso de rehabilitación mediante la inclusión y la vivencia en una estructura social de convivencia, bajo normas, límites, y responsabilidades establecidas, con una distribución clara en sectores y roles de trabajo y funcionamiento, con unos horarios y hábitos de la vida cotidiana establecidos y mantenidos en el tiempo, sumados a la vivencia en valores y la transferencia cultural que supone un proceso de rehumanización de mayor o menor duración.

A partir de los esbozos de De Leon (2004), los procesos de transformación se desarrollan gracias al concepto de "comunidad", entendida como contexto y como método. Contexto porque tiene similares condiciones de un vecindario o residencia, tiene propósitos, estructura organizativa, reglas, normas, valores culturales y creencias; entre el personal y los residentes se genera afiliación, encuentro y convivencia. Y como método desarrollan una serie de actividades que posibilitan el aprendizaje social y la modificación de actitudes, conductas y hábitos para la construcción de nuevos estilos de vida.

Lo anterior sustenta que desde el modelo holístico y el método educativo - terapéutico, la CT se constituye en una estructura social de deshabituación y reeducación de los residentes el cual procura la transformación de sus estilos de vida, de hecho, se presenta un modelo cultural de vida y un juego de roles que permiten ser vivenciados en cualquier otra estructura.

\section{CONCLUSIONES}

La comunidad terapéutica se constituye en un espacio que permite la resocialización gradual del residente a partir del reconocimiento personal, el autocontrol, el mejoramiento de sus relaciones interpersonales e intrafamiliares y favorece el desarrollo de habilidades desde el aprendizaje social, que en palabras de De león (2004), Comas (2010) y Molina (2011), este modelo de comunidad terapéutica sigue siendo una de las estrategias más pertinentes para la reeducación frente a los problemas de farmacodependencia.

El componente terapéutico sustentado en rutinas, confesiones y confrontaciones restablece las relaciones consigo mismo, con su familia y con los otros, facilita la expresión de emociones, aumenta la autoestima, el autocontrol y la responsabilidad, siendo estos protagonistas de su propio tratamiento, proceso dado mediante modelos, roles, 
desarrollo de actividades y vivencia de valores que en palabras de De León (2004), les proporciona las oportunidades y el contexto para asumir una vida social positiva.

La atención terapéutica educativa de esta $\mathrm{CT}$, tiene como propósito promover el desarrollo integral y la restitución de los derechos del adolescente con problemas de adicción, proceso sustentado en las rutinas, el aprendizaje social y la autoayuda el cual posibilitan su recuperación e inserción social. Tanto el componente terapéutico como educativo se sustenta en la interacción social, la cual promueve aprendizajes significativos $y$ la participación activa de los residentes en su vida cotidiana, permitiendo así su integración al sistema social estructurado.

Para los adolescentes residentes, la comunidad terapéutica se constituye en un espacio de encuentro personal y social a partir de modelos de identificación, el auto control, la vivencia de valores y el desarrollo de tareas específicas; un espacio de convivencia, esparcimiento, descanso y refugio. Los modelos de referencia que se destacan son el hermano mayor, el educador y los compañeros de habitación dentro del hogar. Personas significativas en su proceso de identificación y formación.

Los residentes tienen por lo menos una experiencia significativa en la comunidad, con relación a la amistad y a los vínculos afectivos que se establecen dado por los roles y testimonios de vida de los terapeutas como modelos de vida, motivación y experiencia para el crecimiento y la superación personal.

Durante las etapas, rutinas y actividades los y las residentes logran autoconocimiento, identidad personal, comunicación y confrontación con sus referentes y tutores, interacción social, auto control emocional, disciplina, mejoramiento de actitudes de agresión y la construcción de proyectos de vida personal y social.

Los valores que más se destacan en esta comunidad terapéutica son la responsabilidad, el respeto, la honestidad, el amor responsable y la solidaridad. Al igual los adolescentes manifiestan la necesidad de fortalecer en el diario vivir la humildad, el trabajo en equipo, la comunicación asertiva y la transparencia. Al interior de la comunidad estos valores son importantes porque refuerzan su identidad, su autonomía, la cooperación y el sentido de pertenencia convirtiéndose en grandes expectativas de vida social, de servicio y reconocimiento mutuo.

La formación y el vínculo social se convierten en un eje fundamental para la recuperación del proyecto de vida. Y este a la vez, se convierte en el norte que los y las adolescentes desean lograr al finalizar su proceso. Se desarrolla de manera integral al vincular lo personal, familiar, social, espiritual y educativo, buscando generar identidad, retos y nuevos estilos de vida dado a partir de la vida comunitaria y el sentido de vida que logran restablecer. 


\section{REFERENCIAS}

Agudelo, F. (2004). Comunidad terapéutica para drogodependientes y relación educativa. Acciones e Investigaciones Sociales., 19: 127-144. Recuperado en: http://dialnet.unirioja.es

Amaro, P; Chana, V; Martín, E. (2010). Indicación terapéutica y claves de la derivación desde un centro ambulatorio. En Comas Arnau. (Eds.), La metodología de la comunidad terapéutica (pp.13 41). Madrid: Fundación Atenea Grupo Gid.

Apellániz, A. Gómez, M. Moreno, M. y Muñiz, L. (2010). La intervención educativo-terapéutica para menores con problemas de consumo de drogas en los centros residenciales. En Comas Arnau. (Eds.), La metodología de la comunidad terapéutica (pp.13 - 41). Madrid: Fundación Atenea Grupo Gid.

Aristizábal , C. (2008). Teoría y metodología de la investigación. Guía didáctica y módulo. (F. U. Amigó, Ed.) Recuperado en: http://www.funlam.edu.co/administracion.modulo/NIVEL-06/ TeoriaYMetodologiaDeLalnvestigacion.pdf

Asociación Proyecto Hombre. (2012). Recuperado de: http://proyectohombre.es/

Batista, J. (2009, febrero). La teoría de la identidad, más allá de las comunidades terapéuticas. Documento presentado en la XXIV Conferencia Mundial de Comunidades Terapéuticas. Lima:. Instituto Mundo Libre y WFTC. Recuperado en http://www.mundolibre.org.pe/conferencia/ recursos/files/program_es.pdf

Bernal, H. (2011). ¿Porque los adolescentes consumen drogas? "Poiésis". 11(22): 1-10. Recuperado de http://www.funlam.edu.co/revistas/index.php/poiesis/article/view/218

Bueno, A; Limiñana, A. (2002). Guía Didáctica de psicología de la intervención social en la diplomatura del trabajo social. Alicante: Editorial Club Universitario. Recuperado en: http:// books.google.com.co/

Corporación Colombiana de Comunidades Terapéuticas - FECCOT. (2012). Recuperado en: http:// www.feccot.org/

Comas, D. (2010). La Comunidad terapéutica: una perspectiva metodológica. En Comas Arnau. (Eds.), La metodología de la comunidad terapéutica (pp.13 - 41). Madrid: Fundación Atenea Grupo Gid.

Consuegra, N. (Ed.). (2011). Diccionario de Psicología. (2da. ed., Vol. 1). Bogotá: ECOE Ediciones.

De León, G. (2004). La comunidad terapéutica y las adicciones: teoría, modelo y método. España: Editorial Desclée de Brouwer. [Versión e-libro]

Federación Latinoamericana de comunidades terapéuticas - FLACT. (2009). Recuperado de http:// www.flact.org/quees.htm

Fundación de Ayuda contra la Adicción - FAD. (2012). Recuperado de: http://www.fad.es/Home 
Fundación Hogares Claret. (2001). Proyecto de atención institucional. Medellín.

Fundación Hogares Claret. (2002). Memorias Jornadas de evaluación nacional para directores de programas juveniles. Medellín.

Fundación Hogares Claret. (2012). Recuperado en: http://www.fundacionhogaresclaret.org/

Goti, E. (Junio, 2003). La comunidad terapéutica: breve repaso de su historia y algunas definiciones del modelo. Recuperado en: http://www.ieanet.com/opiniones/121

Goti, E. (2009). La comunidad terapéutica un desafío a las drogas. (4ta ed.). Medellín: Fundación Hogares Claret.

Instituto Nacional sobre el abuso de Drogas (2004). Como prevenir el uso de drogas en los niños y adolescentes. (2da ed.). Departamento de salud y servicios humanos de los Estados Unidos. Recuperado en: http://onsm.ces.edu.co/uploads/files/1144459_RedBook_spanish_Prevenciondrogas-adolescentes_NIDA.pdf

Mejía, G. (2000). Memorias Fundación Hogares Claret. Medellín: Fundación Hogares Claret.

Mejía, G. (s. f.). ABC de la Comunidad terapéutica. Medellín: Fundación Hogares Claret.

Molina, A. (2011). El método de comunidad terapéutica para drogodependientes: un análisis desde las ciencias sociales. Adicción y Ciencia, 1(4). Recuperado en: http://dialnet.unirioja.es

Olivar, A. (2010). La educación social en la comunidad terapéutica otros dispositivos residenciales: Aspectos generales. En Comas Arnau (Eds.), La metodología de la comunidad terapéutica (pp.192 - 221). Madrid: Fundación Atenea Grupo Gid.

Pérez Del Río, F. (2011). En qué cambian los pacientes drogodependientes a los seis meses de tratamiento en la comunidad terapéutica. Adicción y Ciencia. 1(4) 1-13. Recuperado en: http:// dialnet.unirioja.es/servlet/articulo?codigo $=3874112$

Pinzón, J. I. \& Sánchez, A. (2005). Construcción de referentes identitarios en jóvenes que participan en un grupo religioso. (Tesis de pregrado; Pontificia Universidad Javeriana. Facultad de Psicologia). Recuperado en: http://www.javeriana.edu.co/biblos/tesis/psicologia/tesis17.pdf

Ministerio de Salud (2002, 26 de febrero). Resolución 0196 sobre normas técnicas, científicas y administrativas para el funcionamiento de los centros de atención, tratamiento y rehabilitación integral, que prestan servicios de salud a personas con problemas asociados al consumo de sustancias psicoactivas. Recuperado en: http://www.minsalud.gov.co/Normatividad/ RESOLUCI\%C3\%93N\%200196\%20DE\%202002.pdf

Soto, C. (2011). Psicoanálisis aplicado al tratamiento de adicciones en comunidades terapéuticas ¿Posible o imposible? Tesis para optar al título de magister en Psicologia Clínica de Adultos. Santiago: Universidad de Chile. Departamento de Psicologia. Recuperado en: http://www.tesis. uchile.cl/tesis/uchile/2011/cs-soto_c/pdfAmont/cs-soto_c.pdf 